\title{
Diversidade da comunidade de plantas invasoras em sistemas agroflorestais com café em Turrialba, Costa Rica
}

\author{
Marta dos Santos Freire Ricci(1), Elias de Melo Virgínio Filho(2) e Janaina Ribeiro Costa(1)
}

(1)Embrapa Agrobiologia, BR 465, Km 7, CEP 23890-000 Seropédica, RJ. E-mail: marta@cnpab.embrapa.br, janaina@cnpab.embrapa.br (2)Centro Agonómico Tropical de Investigación y Enseñanza, Sede Central, no 7.170, Cartago, 30501 Turrialba, Costa Rica. E-mail: eliasdem@catie.ac.cr

\begin{abstract}
Resumo - O objetivo deste trabalho foi avaliar a diversidade de plantas invasoras, em sistemas agroflorestais com cafeeiros (Coffea arabica L.) e em cafezal a pleno sol, sob manejo orgânico e convencional. Foram avaliados 20 tratamentos no delineamento de blocos ao acaso, em parcelas subdivididas no tempo, com três repetições. Os tratamentos corresponderam à combinação entre sistemas agroflorestais e quatro níveis de manejo, baseados na intensidade das práticas e aplicação de insumos: alto e médio convencional, médio e baixo orgânico. Em julho de 2002 e setembro de 2005 foi realizado o levantamento de plantas invasoras. Determinaram-se a riqueza, abundância, diversidade e eqüitabilidade. Observou-se redução nos valores médios de riqueza, abundância, diversidade e equiitabilidade, em que os menores valores foram encontrados no tratamento com manejo alto convencional. Houve redução na freqüência das trepadeiras, ciperáceas e espécies de folhas estreitas (má cobertura), e aumento das espécies de folhas largas (boa cobertura) e gramíneas. Nas duas épocas, os maiores percentuais de pontos com solo descoberto foram encontrados no tratamento alto convencional, e os menores percentuais foram encontrados em todos os tratamentos com manejo médio e baixo orgânico, e em três dos sete tratamentos com manejo médio convencional. Tais resultados demonstram que onde há aplicação freqüiente de herbicida, a presença de árvores não evita exposição do solo.
\end{abstract}

Termos para indexação: Coffea arabica, cobertura vegetal, diversidade, eqüitabilidade, fitossociologia, sombreamento.

\section{Diversity of weed community in agroforestry systems with coffee in Turrialba, Costa Rica}

\begin{abstract}
The objective of this work was to evaluate the floristic composition of spontaneous species in coffee (Coffea arabica L.) cultivated in full sun and in agroforestry systems, under organic and conventional management. Twenty treatments were evaluated in a randomized complete block design, in a split plot in time, with three replicates. The treatments corresponded to combinations between types of agroforestry systems and four management system levels, based on practice intensity and inputs application: high and medium conventional, medium and low organic management. In July 2002 and September 2005 a spontaneous species survey was carried out. Richness, abundance, diversity and evenness index were determined. Reductions were observed in richness, abundance, diversity, and evenness values, and the smallest ones were found in high conventional treatment. There was reduction in the relative frequency of climbing plants, Cyperaceae, and monocot species (poor cover), and increase in broad-leaf species (good cover) and grasses. In both seasons, the highest percentages of points with bare soil were found in the high conventional treatment, while the lowest percentages were found in all treatments under medium and low organic management, and in three out of the seven treatments under medium conventional management. These results demonstrate that in treatments where herbicides are frequently applied, the presence of trees does not prevent the soil from being exposed.
\end{abstract}

Index terms: Coffea arabica, plant cover, diversity, evenness, phytosociology, shading.

\section{Introdução}

Na Costa Rica, o cultivo do café em sistemas agroflorestais ou arborizados é uma prática muito comum, que visa tornar os cafezais mais sustentáveis e diminuir a dependência de insumos externos. As árvores apresentam um importante papel na dinâmica de nutrientes, o que interfere na fertilidade dos solos sob a influência de suas copas, especialmente quando pertencentes à família das leguminosas, que são capazes de aumentar o aporte de nitrogênio via fixação biológica (Dias et al., 2006). A utilização de espécies arbóreas torna os agroecossistemas mais diversificados, favorece a conservação dos recursos naturais, tais como solo, água e biodiversidade (Altieri et al., 1999; Haggar et al., 2001; Alvarenga \& Martins, 2004), e aumenta o 
armazenamento de carbono e nitrogênio do solo (Payán et al., 2002), o que proporciona maior valor econômico às terras, em conseqüência dos serviços ambientais gerados (Ávila et al., 2001).

As plantas invasoras competem com o cafeeiro por água, energia e nutrientes, e podem ocasionar perdas de 60 a $80 \%$ da produção (Silva et al., 2006); seu controle pode representar cerca de $50 \%$ dos custos de produção, em cafezais orgânicos na América Latina (Soto-Pinto et al., 2002).

O grau de competição exercido pelas invasoras varia com a composição florística e com o tipo e a intensidade de manejo utilizado na área, tais como roçadas, capinas, aplicação de herbicidas, adubações, irrigação, entre outros fatores (Erasmo et al., 2004). Neste sentido, um importante benefício da arborização sobre os cafezais consiste na diminuição da competição exercida pelas espécies invasoras, ao se reduzir sua freqüência e alterar sua composição (Nestel \& Altieri, 1992; Beer et al., 1998), por reduzir a radiação solar incidente sobre o solo (Beer, 1987; Peláez, 2004) e por formar uma camada de serapilheira que constitui uma barreira física (Muschler, 2000), bem como por meio da liberação de compostos alelopáticos no solo, que impedem ou reduzem a germinação (Peláez, 2004).

De acordo com Erasmo et al. (2004), uma vez que as comunidades de plantas infestantes podem variar sua composição florística, em razão do tipo e da intensidade de tratos culturais impostos, o reconhecimento das espécies presentes torna-se fundamental para se entender a dinâmica da flora infestante e para se determinarem programas e estratégias de prevenção e manejo (Yanagizawa \& Maimoni-Rodella, 1999; Figueroa et al., 2002).

O objetivo deste trabalho foi avaliar a comunidade e a diversidade de plantas invasoras, em diferentes sistemas agroflorestais com cafeeiros submetidos a diferentes níveis de manejo orgânico e convencional.

\section{Material e Métodos}

O trabalho foi realizado na Estação Experimental do Centro Agronómico Tropical de Investigación y Enseñanza (CATIE), em Turrialba, Costa Rica (9053'N, $83^{\circ} 40^{\prime} \mathrm{W}$ ), a $600 \mathrm{~m}$ de altitude, em área de 9,2 ha, com: relevo levemente inclinado (aproximadamente 5\%); precipitação média de $2.651 \mathrm{~mm}$ por ano; temperatura de $21,8^{\circ} \mathrm{C}$; umidade relativa do ar de $88 \%$; e radiação solar de $16,9 \mathrm{MJ} \mathrm{m}^{-2}$ por dia. Essa área foi anteriormente cultivada com cana-de-açúcar (Saccharum officinarum).

Foram identificadas as seguintes classes de solos: Aluviais mistos, Podzólico e Cambissolo. As análises químicas e físicas foram realizadas no laboratório de Análises de Solos, Tecidos Vegetais e Água do Catie, segundo DíazRomeú \& Hunter (1978) e Nelson \& Sommer (1996), tendo apresentado as seguintes características: $\mathrm{pH}$ em água: 5,4 a 6,1; matéria orgânica (Walkley Black): 4,2 a 7,8; CTC (extração de $\mathrm{NaCl} 10 \%$ pH 2,5): 31 a $41 \mathrm{cmol} \mathrm{kg}^{-1}$; cálcio: 3,4 a 6,2 cmol kg-1; magnésio: 1,2 a 1,8 cmol kg-1; potássio: 0,4 a 1,1 cmol kg-1 e sódio: 0,05 a 0,08 cmol kg-1 (extraídos com acetato de amônio $1 \mathrm{~N} \mathrm{pH} \mathrm{7);} \mathrm{fósforo:} 44$ a 69\%; ferro: 0,7 a 1,9\% e alumínio trocável: 0,5 a 1,2\% (extraídos com oxalato de amônio); densidade aparente: $1,1 \mathrm{~g} \mathrm{~cm}^{-3}$; areia: 100 a $450 \mathrm{~g} \mathrm{~kg}^{-1}$; silte: 360 a $600 \mathrm{~g} \mathrm{~kg}^{-1}$; e argila: 160 a $440 \mathrm{~g} \mathrm{~kg}^{-1}$.

O experimento teve início em agosto de 2000, com o plantio de mudas de café (Coffea arabica), cultivar Caturra, no espaçamento $2 \times 1 \mathrm{~m}$. Foram avaliados 20 tratamentos, dispostos no delineamento de blocos ao acaso, em esquema de parcelas subdivididas no tempo (época de amostragem), com três repetições. Os tratamentos corresponderam à combinação entre sete tipos de sistemas de cultivo de café e quatro níveis de manejo, baseados na intensidade das práticas e aplicação de insumos, denominados manejo alto convencional (AC), médio convencional (MC), médio orgânico (MO) e baixo orgânico (BO). Os sete sistemas de cultivo utilizados foram: café cultivado com Erythrina poeppigiana (E) - eritrina, leguminosa; café com Terminalia amazonia $(\mathrm{T})$ - terminalia, não leguminosa; Chloroleucon eurycyclum (C) - cashá, leguminosa; café com T. amazonia e $C$. eurycyclum (TC); café com E. poeppigiana e T. amazonia (ET); café com E. poeppigiana e $C$. eurycyclum (CE); e café cultivado a pleno sol (PS). Não foi avaliada uma testemunha absoluta, porém foram considerados como testemunha, os dois tratamentos em que o cafeeiro foi cultivado a pleno sol (Tabela 1). O plantio das espécies arbóreas foi feito no espaçamento $6 \mathrm{~m}$ (entre as linhas de plantio) por $4 \mathrm{~m}$ (dentro da mesma linha), em parcelas com tamanho médio de $1.056 \mathrm{~m}^{2}$.

O manejo alto convencional correspondeu à aplicação dos níveis mais altos de insumos e fertilizantes químicos, utilizados pelos cafeicultores na região estudada (300 kg ha-1 por ano de $\mathrm{N}$ e $150 \mathrm{~kg} \mathrm{ha}^{-1}$ por ano de $\mathrm{K}_{2} \mathrm{O}$ ). As quantidades de fertilizantes aplicadas objetivaram 
proporcionar o aporte de nutrientes em quantidades mais elevadas do que as retiradas pelas colheitas. $\mathrm{O}$ controle de pragas e doenças foi feito com base na aplicação de defensivos químicos, tendo-se seguido um calendário de aplicação. O controle de invasoras foi feito por meio de herbicida (sete aplicações por ano) não seletivo, sistêmico, pós-emergente à base de glifosato, tendo-se deixado o solo descoberto.

No manejo médio convencional, foram utilizados níveis intermediários de fertilizantes e insumos, em relação aos utilizados no AC. As quantidades de fertilizantes aplicadas foram elevadas, porém menores que as do manejo AC ( $150 \mathrm{~kg} \mathrm{ha}^{-1}$ por ano de $\mathrm{N} \mathrm{e} 75 \mathrm{~kg} \mathrm{ha}^{-1}$ por ano de $\mathrm{K}_{2} \mathrm{O}$ ). $\mathrm{O}$ controle de pragas e doenças também foi feito com base na aplicação de defensivos químicos, porém realizado menos frequientemente, isto é, não seguiu o mesmo calendário do manejo AC. Foram utilizadas práticas manuais de controle das invasoras, associadas a aplicações menos freqüientes de herbicida (glifosato) nas entrelinhas (cinco aplicações por ano), e capinas completas da linha de plantio do café.

No manejo médio orgânico, foram utilizados níveis intermediários de insumos e fertilizantes, bem como de práticas de manejo, sem, contudo, se correlacionar com o aporte de nutrientes do manejo convencional. Foram aplicados esterco de galinha (10 $\mathrm{Mg} \mathrm{ha}^{-1}$ por ano) e rocha moída (100 kg ha-1 por ano) como fontes de $\mathrm{P}, \mathrm{Ca}$ e $\mathrm{Mg}$, para se devolverem quantidades maiores de nutrientes do que as retiradas pelas colheitas. $\mathrm{O}$ controle de pragas e doenças foi feito com aplicações foliares de produtos naturais e práticas manuais.

No manejo baixo orgânico, utilizaram-se $7 \mathrm{Mg} \mathrm{ha}^{-1}$ por ano de esterco de galinha como fertilizante, sem aplicações de rocha moída como fonte de demais nutrientes, em quantidades que corresponderam às retiradas pelas colheitas. Para o controle de pragas e doenças, não foi utilizado nenhum produto natural, apenas a retirada dos frutos dos cafeeiros no final da colheita para se evitar a broca-do-café (Hypothenemus hampei).

Tanto no MO como no BO, o controle de invasoras foi feito de oito a nove vezes por ano, com o auxílio de roçadeiras costais, tendo-se roçado de forma seletiva nas entrelinhas e capinado nas linhas.

Em julho de 2002 e setembro de 2005, na estação chuvosa, foi realizado o levantamento das espécies de plantas invasoras, pelo método "ponta do sapato", adotado pelo Catie (Staver, 2001), que consistiu em se caminhar em linha reta em dez entrelinhas do cafeeiro por parcela. Em cada entrelinha, foram avaliados dez pontos de leitura, no total de 100 pontos por parcela. Entre cada ponto, contaram-se dez passos, anotou-se a espécie encontrada no centro da ponta do sapato (diâmetro de um lápis), tendo-se contabilizado também os pontos com solo descoberto e com serapilheira. As espécies invasoras foram identificadas e posteriormente separadas por grupo, tendo-se somado as freqüências individuais, e as freqüências totais do agrupamento foram lançadas em tabelas pelo critério de serem ou não desejáveis aos sistemas, e por esta razão, foram denominadas, respectivamente, como boa cobertura e má cobertura. As espécies foram agrupadas em: trepadeiras ou cipós, a maioria considerada indesejável nos cultivos; espécies do gênero Cyperus, consideradas de má cobertura; espécies de folhas largas rasteiras, com altura menor que $12 \mathrm{~cm}$ e com raízes pouco profundas, boa cobertura; espécies de folhas largas indesejáveis, má cobertura; espécies com folhas estreitas consideradas boa cobertura; gramíneas, a maioria indesejável em um manejo seletivo; e outras espécies.

Em seguida, determinou-se a riqueza (número de espécies), a abundância (número total de indivíduos da amostra), a freqüência relativa $(\mathrm{F})$ das espécies, o índice

Tabela 1. Número e código dos tratamentos, constituídos pela combinação entre os tipos de cobertura arbórea para sombreamento do cafeeiro e os níveis de intensidade de manejo do sistema.

\begin{tabular}{|c|c|c|}
\hline Tipo de cobertura arbórea & Nível de manejo $^{(1)}$ & $\begin{array}{c}\text { Número e código } \\
\text { do tratamento }\end{array}$ \\
\hline \multirow{4}{*}{$\begin{array}{l}\text { Erythrina poeppigiana }(\mathrm{E}), \\
\text { eritrina }\end{array}$} & $\mathrm{AC}, \mathrm{MC}, \mathrm{MO}, \mathrm{BO}$ & $1(\mathrm{E}-\mathrm{AC})$ \\
\hline & & 2 (E-MC) \\
\hline & & 3 (E-MO) \\
\hline & & $4(\mathrm{E}-\mathrm{BO})$ \\
\hline \multirow{4}{*}{$\begin{array}{l}\text { Terminalia amazonia }(\mathrm{T}) \text {, } \\
\text { terminalia }\end{array}$} & $\mathrm{AC}, \mathrm{MC}, \mathrm{MO}, \mathrm{BO}$ & $5(\mathrm{~T}-\mathrm{AC})$ \\
\hline & & $6(\mathrm{~T}-\mathrm{MC})$ \\
\hline & & 7 (T-MO) \\
\hline & & $8(\mathrm{~T}-\mathrm{BO})$ \\
\hline \multirow{2}{*}{$\begin{array}{l}\text { Chloroleucon eurycyclum (C), } \\
\text { cashá }\end{array}$} & $\mathrm{MC}, \mathrm{MO}$ & 9 (C-MC) \\
\hline & & $10(\mathrm{C}-\mathrm{MO})$ \\
\hline \multirow[t]{2}{*}{ Terminalia + cashá (TC) } & $\mathrm{MC}, \mathrm{MO}$ & 11 (TC-MC) \\
\hline & & 12 (TC-MO) \\
\hline \multirow[t]{2}{*}{ Eritrina + terminalia $(\mathrm{ET})$} & $\mathrm{MC}, \mathrm{MO}$ & 13 (TE-MC) \\
\hline & & 14 (TE-MO) \\
\hline \multirow[t]{4}{*}{ Eritrina + cashá $(\mathrm{EC})$} & $\mathrm{AC}, \mathrm{MC}, \mathrm{MO}, \mathrm{BO}$ & $15(\mathrm{CE}-\mathrm{AC})$ \\
\hline & & 16 (CE-MC) \\
\hline & & 17 (CE-MO) \\
\hline & & 18 (CE-BO) \\
\hline \multirow[t]{2}{*}{ Café a pleno sol (PS) } & $\mathrm{AC}, \mathrm{MC}$ & 19 (PS-AC) \\
\hline & & 20 (PS-MC) \\
\hline
\end{tabular}

(1) AC: alto convencional; MC: médio convencional; MO: médio orgânico; BO: baixo orgânico. 
de diversidade de Shannon (H') e o índice de eqüitabilidade de Pielou (J'). $\mathrm{O}$ valor de $\mathrm{F}$ foi calculado pela equação $\mathrm{F}=\left(\mathrm{F}_{\mathrm{o}} / \mathrm{F}_{1}\right) 100$, em que: $\mathrm{F}_{\mathrm{o}}$ é a freqüência da espécie e $F_{1}$ é a freqüência total das espécies (Silva et al., 2006). A diversidade de Shannon foi calculada pela equação: $H^{\prime}=-\sum \ln$ pi, em que: $p i=n / N$ (Magurran, 1988); n é o número de indivíduos de uma determinada espécie; e $\mathrm{N}$ é o número total de indivíduos ocorrentes. A eqüitabilidade foi calculada pela expressão: J' = H'/ln S (Martins \& Santos, 1999), em que: S é a riqueza.

A análise de variância dos dados, com aplicação do teste $\mathrm{F}$, e a comparação de médias por meio do teste Scott-Knott, a 5\% de probabilidade, foram feitas pelo programa SISVAR (Ferreira, 2000). Os dados originais das variáveis espécies de folhas estreitas (boa cobertura), gramíneas (a maioria indesejáveis), outras espécies de invasoras e percentagem de pontos amostrados com solo descoberto foram transformadas para arc sen $(\mathrm{x} / 100)^{0,5}$, para as análises estatísticas. Para a construção dos dendrogramas foi utilizado o programa
NTSys 2.1 (Rohlf, 2000) e, como medida de dissimilaridade, foram utilizados a distância euclidiana, indicada para dados quantitativos, e o método de agrupamento UPGMA ("unweighted pair group method with arithmetic mean").

\section{Resultados e Discussão}

Na primeira avaliação das espécies invasoras, realizada em 2002, dois anos após o estabelecimento do experimento, o valor da riqueza, considerando-se a média geral dos tratamentos, foi maior (15,4 espécies) do que o valor encontrado na segunda avaliação, realizada em 2005 (9,9 espécies), assim como o valor da abundância total (número de indivíduos por espécie) foi de 83,9 indivíduos em 2002, em comparação a 57,2 indivíduos em 2005 (Tabela 2). As reduções ocorridas nos valores de riqueza e abundância das espécies, entre as duas épocas, variaram com o tipo de cobertura arbórea e com a intensidade de manejo utilizado (práticas e aplicação de insumos), porém os menores valores foram obtidos nos tratamentos com

Tabela 2. Valores médios de riqueza e abundância (em 100 pontos amostrados) e índice de Shannon (H') e de eqüitabilidade de Pielou (J') dos tratamentos, em julho de 2002 e setembro de $2005^{(1)}$.

\begin{tabular}{|c|c|c|c|c|c|c|c|c|}
\hline \multirow{2}{*}{ Tratamento $^{(2)}$} & \multicolumn{2}{|c|}{ Riqueza } & \multicolumn{2}{|c|}{ Abundância } & \multicolumn{2}{|c|}{ Índice de Shannon (H') } & \multicolumn{2}{|c|}{ Eqüitabilidade (J') } \\
\hline & 2002 & 2005 & 2002 & 2005 & 2002 & 2005 & 2002 & 2005 \\
\hline & \multicolumn{8}{|c|}{ Alto convencional } \\
\hline $1(\mathrm{E}-\mathrm{AC})$ & $6,0 \mathrm{Ac}$ & $3,3 \mathrm{Ac}$ & $13,7 \mathrm{Ac}$ & $8,7 \mathrm{Ac}$ & $1,56 \mathrm{Ab}$ & $0,72 \mathrm{Bb}$ & $0,88 \mathrm{Aa}$ & $0,49 \mathrm{Ba}$ \\
\hline $5(\mathrm{~T}-\mathrm{AC})$ & $10,7 \mathrm{Ab}$ & $1,0 \mathrm{Bc}$ & $33,7 \mathrm{Ab}$ & $3,3 \mathrm{Bc}$ & $1,87 \mathrm{Ab}$ & $0,00 \mathrm{Bc}$ & $0,80 \mathrm{Aa}$ & $0,00 \mathrm{Bc}$ \\
\hline $15(\mathrm{CE}-\mathrm{AC})$ & $8,0 \mathrm{Ac}$ & $2,3 \mathrm{Bc}$ & $18,0 \mathrm{Ac}$ & $4,7 \mathrm{Ac}$ & $1,64 \mathrm{Ab}$ & $0,69 \mathrm{Bb}$ & $0,82 \mathrm{Aa}$ & $0,56 \mathrm{Ba}$ \\
\hline \multirow[t]{2}{*}{19 (PS-AC) } & $10,7 \mathrm{Ab}$ & $3,0 \mathrm{Bc}$ & $48,0 \mathrm{Ab}$ & $18,0 \mathrm{Bc}$ & $2,04 \mathrm{Ab}$ & $0,53 \mathrm{Bb}$ & $0,87 \mathrm{Aa}$ & $0,27 \mathrm{Bb}$ \\
\hline & \multicolumn{8}{|c|}{ Médio convencional } \\
\hline 2 (E-MC) & $18,3 \mathrm{Aa}$ & $9,7 \mathrm{Bb}$ & $88,7 \mathrm{Aa}$ & $23,3 \mathrm{Bb}$ & $2,50 \mathrm{Aa}$ & $1,77 \mathrm{Ba}$ & $0,86 \mathrm{Aa}$ & $0,79 \mathrm{Aa}$ \\
\hline $6(\mathrm{~T}-\mathrm{MC})$ & $16,3 \mathrm{Aa}$ & $15,3 \mathrm{Aa}$ & $100,0 \mathrm{Aa}$ & $83,0 \mathrm{Ba}$ & $2,33 \mathrm{Aa}$ & $1,76 \mathrm{Ba}$ & $0,84 \mathrm{Aa}$ & $0,63 \mathrm{Aa}$ \\
\hline 9 (C-MC) & $18,0 \mathrm{Aa}$ & $15,3 \mathrm{Aa}$ & $97,0 \mathrm{Aa}$ & $83,3 \mathrm{Aa}$ & $2,50 \mathrm{Aa}$ & $1,96 \mathrm{Aa}$ & $0,87 \mathrm{Aa}$ & $0,72 \mathrm{Aa}$ \\
\hline 11 (TC-MC) & $19,3 \mathrm{Aa}$ & $15,0 \mathrm{Aa}$ & $99,0 \mathrm{Aa}$ & $73,7 \mathrm{Ba}$ & $2,45 \mathrm{Aa}$ & $2,13 \mathrm{Aa}$ & $0,83 \mathrm{Aa}$ & $0,79 \mathrm{Aa}$ \\
\hline 13 (TE-MC) & $16,7 \mathrm{Aa}$ & $10,3 \mathrm{Bb}$ & $98,3 \mathrm{Aa}$ & $29,7 \mathrm{Bb}$ & $2,31 \mathrm{Aa}$ & $1,88 \mathrm{Aa}$ & $0,82 \mathrm{Aa}$ & $0,84 \mathrm{Aa}$ \\
\hline 16 (CE-MC) & $18,3 \mathrm{Aa}$ & $9,3 \mathrm{Bb}$ & $96,3 \mathrm{Aa}$ & $43,7 \mathrm{Bb}$ & $2,17 \mathrm{Aa}$ & $1,77 \mathrm{Aa}$ & $0,75 \mathrm{Aa}$ & $0,79 \mathrm{Aa}$ \\
\hline \multirow[t]{2}{*}{20 (PS-MC) } & $22,3 \mathrm{Aa}$ & $11,3 \mathrm{Bb}$ & $97,3 \mathrm{Aa}$ & $72,3 \mathrm{Ba}$ & $2,57 \mathrm{Aa}$ & $1,62 \mathrm{Ba}$ & $0,84 \mathrm{Aa}$ & $0,66 \mathrm{Aa}$ \\
\hline & \multicolumn{8}{|c|}{ Médio orgânico } \\
\hline 3 (E-MO) & $13,7 \mathrm{Ab}$ & $7,3 \mathrm{Bc}$ & $96,0 \mathrm{Aa}$ & $39,7 \mathrm{Bb}$ & $2,26 \mathrm{Aa}$ & $1,21 \mathrm{Aa}$ & $0,87 \mathrm{Aa}$ & $0,61 \mathrm{Ba}$ \\
\hline 7 (T-MO) & $17,3 \mathrm{Aa}$ & $14,3 \mathrm{Aa}$ & $99,3 \mathrm{Aa}$ & $77,3 \mathrm{Ba}$ & $2,39 \mathrm{Aa}$ & $2,08 \mathrm{Aa}$ & $0,84 \mathrm{Aa}$ & $0,78 \mathrm{Aa}$ \\
\hline $10(\mathrm{C}-\mathrm{MO})$ & $17,7 \mathrm{Aa}$ & $11,0 \mathrm{Bb}$ & $100,0 \mathrm{Aa}$ & $96,7 \mathrm{Aa}$ & $2,40 \mathrm{Aa}$ & $1,89 \mathrm{Aa}$ & $0,83 \mathrm{Aa}$ & $0,79 \mathrm{Aa}$ \\
\hline 12 (TC-MO) & $16,3 \mathrm{Aa}$ & $12,7 \mathrm{Aa}$ & $97,0 \mathrm{Aa}$ & $87,3 \mathrm{Aa}$ & $2,36 \mathrm{Aa}$ & $2,02 \mathrm{Aa}$ & $0,85 \mathrm{Aa}$ & $0,80 \mathrm{Aa}$ \\
\hline 14 (TE-MO) & $20,3 \mathrm{Aa}$ & $11,0 \mathrm{Bb}$ & $98,7 \mathrm{Aa}$ & $72,7 \mathrm{Ba}$ & $2,62 \mathrm{Aa}$ & $1,74 \mathrm{Ba}$ & $0,87 \mathrm{Aa}$ & $0,73 \mathrm{Aa}$ \\
\hline \multirow[t]{2}{*}{17 (CE-MO) } & $14,7 \mathrm{Aa}$ & $12,3 \mathrm{Aa}$ & $98,3 \mathrm{Aa}$ & $79,0 \mathrm{Aa}$ & $2,20 \mathrm{Aa}$ & $1,76 \mathrm{Aa}$ & $0,82 \mathrm{Aa}$ & $0,71 \mathrm{Aa}$ \\
\hline & \multicolumn{8}{|c|}{ Baixo orgânico } \\
\hline $4(\mathrm{E}-\mathrm{BO})$ & $13,3 \mathrm{Ab}$ & $9,7 \mathrm{Ab}$ & $100,0 \mathrm{Aa}$ & $69,0 \mathrm{Ba}$ & $2,10 \mathrm{Aa}$ & $1,49 \mathrm{Aa}$ & $0,81 \mathrm{Aa}$ & $0,66 \mathrm{Aa}$ \\
\hline $8(\mathrm{~T}-\mathrm{BO})$ & $15,0 \mathrm{Aa}$ & $14,7 \mathrm{Aa}$ & $98,3 \mathrm{Aa}$ & $97,3 \mathrm{Aa}$ & $2,28 \mathrm{Aa}$ & $1,96 \mathrm{Aa}$ & $0,85 \mathrm{Aa}$ & $0,73 \mathrm{Aa}$ \\
\hline $18(\mathrm{CE}-\mathrm{BO})$ & $15,7 \mathrm{Aa}$ & $8,7 \mathrm{Bb}$ & $99,0 \mathrm{Aa}$ & $82,0 \mathrm{Aa}$ & $2,37 \mathrm{Aa}$ & $1,38 \mathrm{Ba}$ & $0,86 \mathrm{Aa}$ & $0,66 \mathrm{Ba}$ \\
\hline Média & $15,4 \mathrm{~A}$ & $9,9 \mathrm{~B}$ & $83,9 \mathrm{~A}$ & $57,2 \mathrm{~B}$ & $2,25 \mathrm{~A}$ & $1,52 \mathrm{~B}$ & $0,84 \mathrm{~A}$ & $0,65 \mathrm{~B}$ \\
\hline $\mathrm{CV}$ tratamentos $(\%)$ & \multicolumn{2}{|c|}{19,2} & \multicolumn{2}{|c|}{12,1} & \multicolumn{2}{|c|}{19,4} & \multicolumn{2}{|c|}{23,9} \\
\hline CV época $(\%)$ & \multicolumn{2}{|c|}{25,3} & \multicolumn{2}{|c|}{17,9} & \multicolumn{2}{|c|}{17,9} & \multicolumn{2}{|c|}{16,7} \\
\hline
\end{tabular}


manejo alto convencional (AC), em que o controle das invasoras foi feito com herbicida não seletivo.

O mesmo comportamento foi observado em relação à diversidade espacial ou heterogeneidade, expressa pelo índice de Shannon, isto é, independentemente do manejo, a média dos tratamentos apresentou redução de H'de 2,25 em 2002 para 1,52 em 2005 (Tabela 2). Em relação aos valores de referência citados por Cavalcanti \& Larrazábal (2004), que consideraram a diversidade de Shannon alta quando está acima de 3 , média entre 2 e 3 , baixa entre 1 e 2 e muito baixa quando menor que 1 , os valores médios de diversidade encontrados no presente trabalho foram de médios a baixos. De acordo com o nível de manejo, os valores de diversidade encontrados nos tratamentos com manejo AC não foram significativamente diferentes entre si (Tabela 2), tendo variado entre 1,56 e 2,04 (diversidade baixa a média) em 2002, e entre 0 e 0,72 (muito baixa) em 2005. Os tratamentos com manejo MC não apresentaram diferenças entre si, em nenhuma das épocas avaliadas, e os valores variaram entre 2,17 e 2,57 (média) em 2002, e entre 1,62 e 2,13 (de baixa a média) em 2005. Da mesma forma, não foram observadas diferenças significativas entre os tratamentos com os manejos MO e BO; no manejo MO, os valores variaram de 2,20 a 2,62 (média) em 2002, e entre 1,21 e 2,08 (de baixa a média) em 2005, e no manejo BO, de 2,10 a 2,37 (média) em 2002, e entre 1,38 e 1,96 (baixa) em 2005. Portanto, os menores valores de diversidade, em ambas as épocas avaliadas, foram encontrados nos tratamentos AC, que foram significativamente menores em relação aos demais tratamentos.

As reduções na diversidade (média dos tratamentos) observadas neste trabalho estão de acordo com estudo realizado por Silva et al. (2006), em que seis cafezais arborizados com diferentes densidades de plantio de Grevillea robusta foram comparados, em relação ao cultivo a pleno sol no Sudoeste da Bahia, Brasil, entre abril de 2004 e março de 2005. Os autores concluíram que o valor médio de diversidade (médias dos tratamentos), que em abril de 2004 variou de 0 a 3,5, foi de 0,6 a 1,2 em março de 2005. Entretanto, a diversidade de espécies invasoras, determinada pelo índice de Shannon, foi maior nos cafezais arborizados, em que maior número de espécies de plantas invasoras dicotiledôneas foram identificadas.

A eqüitabilidade, que expressa o grau de distribuição das espécies dentro de uma comunidade, avaliada pelo índice de Pielou, também diminuiu ao longo do período avaliado. De acordo com Cavalcanti \& Larrazábal (2004), a eqüitabilidade varia entre 0 e 1 , e é considerada alta quando maior que 0,5 . De acordo com esses autores, um baixo valor de eqüitabilidade significa que existe a dominância de uma ou mais espécies na comunidade estudada, enquanto eqüitabilidade alta significa distribuição uniforme entre as espécies na amostra e demonstra que, apesar da complexidade da comunidade, os indivíduos encontram-se bem distribuídos. A equiitabilidade alta também explica a estabilidade ambiental, o que, segundo Levinton (1995), significa pequenas amplitudes nas variáveis ambientais em curto prazo.

Entre os tratamentos com manejo AC, a eqüitabilidade variou entre 0,80 e 0,88 (alta) em 2002, não tendo havido diferença significativa entre os tratamentos, e de 0 a 0,56 (de baixa a alta) em 2005 , porém os tratamentos 5 (T-AC) e 19 (PS-AC) apresentaram valores significativamente mais baixos (Tabela 2). Nos tratamentos MC, a variação foi de 0,75 a 0,87 (alta) em 2002 , e de 0,63 a 0,84 (alta) em 2005, não tendo havido diferença significativa entre os tratamentos em nenhuma época avaliada. Nos tratamentos $\mathrm{MO}$, a variação foi de 0,82 a 0,87 (alta) em 2002, e de 0,61 a 0,80 (alta) em 2005 , e não houve diferença significativa entre os tratamentos em nenhuma época. No manejo BO, a equiitabilidade variou de 0,81 a 0,86 (alta) em 2002 , e de 0,61 a 0,80 (alta) em 2005, e também não apresentou diferença significativa entre os tratamentos nas épocas avaliadas. Os valores de eqüitabilidade encontrados na maioria dos tratamentos, nas duas avaliações, foram considerados altos, o que significa que os sistemas estudados apresentaram boa distribuição das espécies invasoras, exceção feita aos tratamentos $\mathrm{AC}$, durante a segunda avaliação, que apresentaram valores de eqüitabilidade significativamente reduzidos.

Em relação à frequiência dos diferentes tipos de invasoras, combase na média dos tratamentos ao longo do período avaliado (Tabela 3), houve redução na frequiência das trepadeiras, de ciperáceas e das espécies de folhas estreitas (boa cobertura), e aumento das espécies de folhas largas (boa cobertura), de gramíneas e de outras espécies de menor ocorrência, não tendo havido alteração na frequiência de espécies de folhas largas (má cobertura).

Em 2002, as espécies consideradas indesejáveis, constituídas por espécies de trepadeiras, folhas largas (má cobertura), ciperáceas e gramíneas, constituíram 93,2\% do total identificado, reduzido para 82,1\% em 2005 (Tabela 3). Em 2002, as espécies consideradas como 
Tabela 3. Frequiências de espécies invasoras, agrupadas de acordo com o critério de boa ou má cobertura, em razão dos tratamentos e das épocas de amostragem (julho de 2002 e setembro de 2005) ${ }^{(1)}$.

\begin{tabular}{|c|c|c|c|c|c|c|c|c|c|c|c|c|c|c|}
\hline \multirow[t]{2}{*}{ Tratamento $^{(2)}$} & \multicolumn{2}{|c|}{$\begin{array}{c}\text { Trepadeiras } \\
\text { (a maioria indesejável) }\end{array}$} & \multicolumn{2}{|c|}{$\begin{array}{c}\text { Ciperáceas } \\
\text { (má cobertura) }\end{array}$} & \multicolumn{2}{|c|}{$\begin{array}{c}\text { Espécies de folhas } \\
\text { largas (boa cobertura) }\end{array}$} & \multicolumn{2}{|c|}{$\begin{array}{l}\text { Espécies de folhas } \\
\text { largas (má cobertura) }\end{array}$} & \multicolumn{2}{|c|}{$\begin{array}{c}\text { Espécies de folhas } \\
\text { estreitas (boa cobertura) }^{(3)} \\
\end{array}$} & \multicolumn{2}{|c|}{$\begin{array}{c}\text { Gramíneas (a maioria } \\
\text { indesejável) }{ }^{(3)} \\
\end{array}$} & \multicolumn{2}{|c|}{ Outras espécies ${ }^{(3)}$} \\
\hline & 2002 & 2005 & 2002 & 2005 & 2002 & 2005 & 2002 & 2005 & 2002 & 2005 & 2002 & 2005 & 2002 & 2005 \\
\hline & \multicolumn{14}{|c|}{ Alto convencional } \\
\hline 1 (E-AC) & $0,0 \mathrm{Ab}$ & $0,0 \mathrm{Aa}$ & $10,7 \mathrm{Ab}$ & $0,0 \mathrm{Aa}$ & $9,5 \mathrm{Aa}$ & $0,0 \mathrm{Ab}$ & $59,2 \mathrm{Bb}$ & $96,5 \mathrm{Aa}$ & $0,0 \mathrm{Ab}$ & $0,0 \mathrm{Aa}$ & $15,8 \mathrm{Aa}$ & $3,5 \mathrm{Ab}$ & $2,4 \mathrm{Aa}$ & $0,0 \mathrm{Aa}$ \\
\hline $5(\mathrm{~T}-\mathrm{AC})$ & $1,4 \mathrm{Ab}$ & $0,0 \mathrm{Aa}$ & $2,6 \mathrm{Ab}$ & $0,0 \mathrm{Aa}$ & $0,0 \mathrm{Aa}$ & $0,0 \mathrm{Ab}$ & $87,1 \mathrm{Aa}$ & $100,0 \mathrm{Aa}$ & $0,0 \mathrm{Ab}$ & $0,0 \mathrm{Aa}$ & $8,8 \mathrm{Aa}$ & $0,0 \mathrm{Ab}$ & $0,0 \mathrm{Aa}$ & $0,0 \mathrm{Aa}$ \\
\hline 15 (CE-AC) & $1,8 \mathrm{Ab}$ & $0,0 \mathrm{Aa}$ & $2,2 \mathrm{Ab}$ & $0,0 \mathrm{Aa}$ & $0,0 \mathrm{Aa}$ & $0,0 \mathrm{Ab}$ & $92,5 \mathrm{Aa}$ & $41,7 \mathrm{Bb}$ & $0,0 \mathrm{Ab}$ & $0,0 \mathrm{Aa}$ & $3,5 \mathrm{Aa}$ & $20,8 \mathrm{Ab}$ & $0,0 \mathrm{Aa}$ & $4,2 \mathrm{Aa}$ \\
\hline \multirow[t]{2}{*}{19 (PS-AC) } & $0,0 \mathrm{Ab}$ & $0,0 \mathrm{Aa}$ & $5,5 \mathrm{Ab}$ & $0,0 \mathrm{Aa}$ & $2,4 \mathrm{Aa}$ & $0,0 \mathrm{Ab}$ & $73,6 \mathrm{Aa}$ & $94,7 \mathrm{Aa}$ & $1,6 \mathrm{Aa}$ & $0,0 \mathrm{Aa}$ & $16,9 \mathrm{Aa}$ & $5,3 \mathrm{Ab}$ & $0,0 \mathrm{Aa}$ & $0,0 \mathrm{Aa}$ \\
\hline & \multicolumn{14}{|c|}{ Médio convencional } \\
\hline 2 (E-MC) & $4,8 \mathrm{Aa}$ & $0,0 \mathrm{Ba}$ & $23,4 \mathrm{Aa}$ & $1,3 \mathrm{Ba}$ & 6,1Aa & $2,8 \mathrm{Ab}$ & $48,6 \mathrm{Ab}$ & $33,8 \mathrm{Ab}$ & $1,4 \mathrm{Aa}$ & $0,0 \mathrm{Ba}$ & $13,6 \mathrm{Ba}$ & $50,9 \mathrm{Aa}$ & $5,3 \mathrm{Aa}$ & $5,9 \mathrm{Aa}$ \\
\hline 6 (T-MC) & $0,3 \mathrm{Ab}$ & $0,0 \mathrm{Aa}$ & 19,0Aa & $0,7 \mathrm{Ba}$ & $1,7 \mathrm{Aa}$ & $2,0 \mathrm{Ab}$ & $60,3 \mathrm{Ab}$ & $35,4 \mathrm{Ab}$ & $1,7 \mathrm{Aa}$ & $0,4 \mathrm{Aa}$ & $17,0 \mathrm{Ba}$ & $51,9 \mathrm{Aa}$ & $0,0 \mathrm{Aa}$ & $13,3 \mathrm{Aa}$ \\
\hline 9 (C-MC) & $3,4 \mathrm{Aa}$ & $0,0 \mathrm{Ba}$ & $16,7 \mathrm{Aa}$ & $0,3 \mathrm{Ba}$ & $5,1 \mathrm{Aa}$ & $5,7 \mathrm{Ab}$ & $64,8 \mathrm{Ab}$ & $36,4 \mathrm{Ab}$ & $1,4 \mathrm{Aa}$ & $0,0 \mathrm{Ba}$ & $8,6 \mathrm{Ba}$ & $44,6 \mathrm{Aa}$ & $0,0 \mathrm{Ba}$ & $12,9 \mathrm{Aa}$ \\
\hline 13 (TE-MC) & $3,1 \mathrm{Aa}$ & $0,0 \mathrm{Aa}$ & $32,4 \mathrm{Aa}$ & $2,9 \mathrm{Ba}$ & $1,7 \mathrm{Aa}$ & $6,3 \mathrm{Ab}$ & $50,9 \mathrm{Ab}$ & $53,0 \mathrm{Ab}$ & $1,4 \mathrm{Aa}$ & $0,0 \mathrm{Ba}$ & $10,9 \mathrm{Ba}$ & $35,3 \mathrm{Aa}$ & $0,0 \mathrm{Aa}$ & $3,0 \mathrm{Aa}$ \\
\hline 11 (TC-MC) & $0,7 \mathrm{Ab}$ & $0,9 \mathrm{Aa}$ & $20,5 \mathrm{Aa}$ & $0,0 \mathrm{Ba}$ & $4,4 \mathrm{Aa}$ & $10,0 \mathrm{Ab}$ & $58,2 \mathrm{Ab}$ & $48,0 \mathrm{Ab}$ & $0,3 \mathrm{Ab}$ & $0,0 \mathrm{Aa}$ & $15,8 \mathrm{Aa}$ & $29,5 \mathrm{Aa}$ & $0,0 \mathrm{Ba}$ & $17,3 \mathrm{Aa}$ \\
\hline 16 (CE-MC) & $3,4 \mathrm{Aa}$ & $0,7 \mathrm{Ba}$ & $16,6 \mathrm{Aa}$ & $0,0 \mathrm{Ba}$ & $1,4 \mathrm{Aa}$ & $6,6 \mathrm{Ab}$ & $53,1 \mathrm{Ab}$ & $36,3 \mathrm{Ab}$ & $0,7 \mathrm{Aa}$ & $0,0 \mathrm{Aa}$ & $23,6 \mathrm{Ba}$ & $48,2 \mathrm{Aa}$ & $0,7 \mathrm{Aa}$ & $12,3 \mathrm{Aa}$ \\
\hline \multirow[t]{2}{*}{20 (PS-MC) } & $0,7 \mathrm{Ab}$ & $0,7 \mathrm{Aa}$ & $13,1 \mathrm{Ab}$ & $0,0 \mathrm{Aa}$ & $4,4 \mathrm{Aa}$ & $4,2 \mathrm{Ab}$ & $70,8 \mathrm{Aa}$ & $45,4 \mathrm{Ab}$ & $1,0 \mathrm{Aa}$ & $0,0 \mathrm{Aa}$ & $10,0 \mathrm{Ba}$ & $43,5 \mathrm{Aa}$ & $0,0 \mathrm{Aa}$ & $6,2 \mathrm{Aa}$ \\
\hline & \multicolumn{14}{|c|}{ Médio orgânico } \\
\hline 3 (E-MO) & $0,0 \mathrm{Ab}$ & $0,0 \mathrm{Aa}$ & $21,2 \mathrm{Aa}$ & $0,0 \mathrm{Ba}$ & $10,4 \mathrm{Aa}$ & $21,7 \mathrm{Aa}$ & $50,9 \mathrm{Ab}$ & $63,5 \mathrm{Ab}$ & $0,0 \mathrm{Ab}$ & $0,0 \mathrm{Aa}$ & $17,4 \mathrm{Aa}$ & $10,4 \mathrm{Ab}$ & $0,0 \mathrm{Aa}$ & $4,3 \mathrm{Aa}$ \\
\hline 7 (T-MO) & $1,6 \mathrm{Ab}$ & $0,4 \mathrm{Aa}$ & $20,8 \mathrm{Aa}$ & $3,7 \mathrm{Ba}$ & $5,0 \mathrm{Aa}$ & $19,7 \mathrm{Aa}$ & $42,7 \mathrm{Ab}$ & $35,7 \mathrm{Ab}$ & $0,0 \mathrm{Ab}$ & $0,0 \mathrm{Aa}$ & $28,3 \mathrm{Aa}$ & $47,4 \mathrm{Aa}$ & $1,6 \mathrm{Aa}$ & $3,7 \mathrm{Aa}$ \\
\hline 10 (C-MO) & $0,0 \mathrm{Ab}$ & $0,6 \mathrm{Aa}$ & $27,1 \mathrm{Aa}$ & $0,8 \mathrm{Ba}$ & $10,0 \mathrm{Ba}$ & $32,7 \mathrm{Aa}$ & $41,6 \mathrm{Ab}$ & $39,7 \mathrm{Ab}$ & $0,0 \mathrm{Ab}$ & $0,0 \mathrm{Aa}$ & $21,3 \mathrm{Aa}$ & $20,0 \mathrm{Aa}$ & $0,0 \mathrm{Aa}$ & $7,8 \mathrm{Aa}$ \\
\hline 12 (TC-MO) & $1,3 \mathrm{Ab}$ & $0,6 \mathrm{Aa}$ & $22,6 \mathrm{Aa}$ & $0,0 \mathrm{Ba}$ & $10,6 \mathrm{Aa}$ & $24,8 \mathrm{Aa}$ & $47,6 \mathrm{Ab}$ & $29,6 \mathrm{Ab}$ & $0,0 \mathrm{Ab}$ & $0,0 \mathrm{Aa}$ & $17,9 \mathrm{Aa}$ & $38,0 \mathrm{Aa}$ & $0,0 \mathrm{Aa}$ & $8,1 \mathrm{Aa}$ \\
\hline 14 (TE-MO) & $2,7 \mathrm{Aa}$ & $1,7 \mathrm{Aa}$ & $20,8 \mathrm{Aa}$ & $3,2 \mathrm{Ba}$ & $6,0 \mathrm{Aa}$ & $14,7 \mathrm{Ab}$ & $54,7 \mathrm{Ab}$ & $58,4 \mathrm{Ab}$ & $0,7 \mathrm{Aa}$ & $0,0 \mathrm{Aa}$ & $15,8 \mathrm{Aa}$ & $24,8 \mathrm{Aa}$ & $0,3 \mathrm{Aa}$ & $7,6 \mathrm{Aa}$ \\
\hline \multirow[t]{2}{*}{17 (CE-MO) } & $0,0 \mathrm{Ab}$ & $0,4 \mathrm{Aa}$ & $17,2 \mathrm{Aa}$ & $1,9 \mathrm{Ba}$ & $13,5 \mathrm{Ba}$ & $38,0 \mathrm{Aa}$ & $50,5 \mathrm{Ab}$ & $51,8 \mathrm{Ab}$ & $0,3 \mathrm{Ab}$ & $0,0 \mathrm{Aa}$ & $16,3 \mathrm{Aa}$ & $8,7 \mathrm{Ab}$ & $1,3 \mathrm{Aa}$ & $4,1 \mathrm{Aa}$ \\
\hline & \multicolumn{14}{|c|}{ Baixo orgânico } \\
\hline 4 (E-BO) & $0,0 \mathrm{Ab}$ & $0,0 \mathrm{Aa}$ & $31,1 \mathrm{Aa}$ & $0,7 \mathrm{Ba}$ & $7,8 \mathrm{Aa}$ & $19,1 \mathrm{Aa}$ & $37,4 \mathrm{Ab}$ & $57,4 \mathrm{Ab}$ & $0,0 \mathrm{Ab}$ & 0,0 & $23,7 \mathrm{Aa}$ & $35,1 \mathrm{Aa}$ & $0,0 \mathrm{Aa}$ & $0,0 \mathrm{Aa}$ \\
\hline 8 (T-BO) & $1,7 \mathrm{Ab}$ & $0,0 \mathrm{Aa}$ & $26,1 \mathrm{Aa}$ & $3,0 \mathrm{Ba}$ & $8,0 \mathrm{Ba}$ & $27,9 \mathrm{Aa}$ & $47,3 \mathrm{Ab}$ & $28,44 \mathrm{Ab}$ & $1,0 \mathrm{Aa}$ & $0,0 \mathrm{Aa}$ & $15,2 \mathrm{Ba}$ & $44,2 \mathrm{Aa}$ & $0,7 \mathrm{Aa}$ & $0,0 \mathrm{Aa}$ \\
\hline 18 (CE-BO) & $2,0 \mathrm{Aa}$ & $0,0 \mathrm{Aa}$ & $24,3 \mathrm{Aa}$ & $0,6 \mathrm{Ba}$ & $31,1 \mathrm{Aa}$ & $6,4 \mathrm{Ba}$ & $50,8 \mathrm{Ab}$ & $58,6 \mathrm{Ab}$ & $0,3 \mathrm{Ab}$ & $0,0 \mathrm{Aa}$ & $16,2 \mathrm{Aa}$ & $10,3 \mathrm{Ab}$ & $0,0 \mathrm{Aa}$ & $5,8 \mathrm{Aa}$ \\
\hline Média & $1,5 \mathrm{~A}$ & $0,3 \mathrm{~B}$ & $18,8 \mathrm{~A}$ & $1,0 \mathrm{~B}$ & $5,6 \mathrm{~B}$ & $12,1 \mathrm{~A}$ & $57,1 \mathrm{~A}$ & $52,2 \mathrm{~A}$ & $0,6 \mathrm{~A}$ & $0,0 \mathrm{~B}$ & $15,8 \mathrm{~B}$ & $28,6 \mathrm{~A}$ & $0,6 \mathrm{~B}$ & $5,8 \mathrm{~A}$ \\
\hline \multirow{2}{*}{\multicolumn{2}{|c|}{ CV tratamentos (\%) }} & & \multirow{2}{*}{\multicolumn{2}{|c|}{$\frac{85,4}{790}$}} & \multirow{2}{*}{\multicolumn{2}{|c|}{$\begin{array}{l}95,9 \\
99,0\end{array}$}} & \multirow{2}{*}{\multicolumn{2}{|c|}{$\begin{array}{l}27,6 \\
380\end{array}$}} & \multirow{2}{*}{\multicolumn{2}{|c|}{$\begin{array}{l}37,4 \\
377\end{array}$}} & \multirow{2}{*}{\multicolumn{2}{|c|}{34,1}} & \multicolumn{2}{|c|}{83,0} \\
\hline & & & & & & & & & & & & & & \\
\hline
\end{tabular}

${ }^{(1)}$ Médias seguidas de letras iguais, minúsculas na coluna (tratamento) e maiúsculas na linha (época), não diferem entre si pelo teste de Scott-Knott, a 5\% de probabilidade. (2)E: eritrina;

T: terminalia; C: cashá; PS: pleno sol; AC: manejo alto convencional; MC: médio convencional; MO: médio orgânico; BO: baixo orgânico. ${ }^{(3)}$ Dados transformados para arc sen (x/100) ${ }^{0,5}$ 
boa cobertura constituíram $6,2 \%$ do total, enquanto em 2005 , este valor aumentou para $12,1 \%$. Espécies com ocorrência rara nos sistemas, classificadas como "outras espécies", que inicialmente totalizaram apenas $0,6 \%$, tiveram a sua freqüência aumentada para 5,8\% em 2005.

Em 2005, a freqüência das espécies de folhas largas (boa cobertura) aumentou significativamente, considerando-se a média dos tratamentos (Tabela 3), em que os aumentos mais significativos foram observados nos tratamentos 10 (C-MO), 17 (CE-MO) e 8 (T-BO). Por sua vez, a frequiência das gramíneas (média dos tratamentos) aumentou, resultado que possivelmente está relacionado ao uso de herbicida nos manejos AC e MC, o que explica as reduções observadas nos tratamentos AC e MC, nos valores de diversidade (H'), e nos valores de eqüitabilidade observados nos tratamentos AC (Tabela 2). A utilização de herbicidas promove a permanência de espécies tolerantes ou resistentes, as quais têm a sua freqüência aumentada por ficar livre da competição de outras espécies (Aguilar et al., 1997).

Alguns trabalhos têm relatado a influência do sombreamento e do tipo de manejo sobre a frequiência e composição das espécies invasoras em cafezais. Nestel \& Altieri (1992) avaliaram sistemas agroflorestais com café no México e observaram que espécies da família Commelinaceae, que competem pouco com o café e são de fácil controle, predominaram nos sistemas arborizados, enquanto no cultivo a pleno sol predominaram famílias de espécies mais agressivas das famílias Poacea e Compositae. Silva et al. (2006) observaram que a arborização de cafezais com Grevillea robusta reduziu a frequiência e aumentou a diversidade de espécies invasoras, tendo sido encontradas 5 espécies monocotiledôneas e 16 espécies dicotiledôneas. SotoPinto et al. (2002) avaliaram a ocorrência de espécies invasoras, em sistemas agroflorestais com café e relataram que $69 \%$ do total de invasoras identificadas foram constituídas por espécies de folhas largas (dicotiledôneas), e $31 \%$ por espécies de folha estreita (em geral monocotiledôneas). Em estudo anterior, Aguilar et al. (1995) concluíram que o manejo convencional, com herbicidas e capina não seletiva, promoveu aumento na frequiência de gramíneas, quando comparado ao controle feito por meio de capinas seletivas.

Dos 20 tratamentos avaliados, 18 foram constituídos por SAFs e apenas 2 a pleno sol (tratamentos 19 e 20). A alteração na freqüência e na composição das espécies invasoras foi mais influenciada pelo tipo de manejo adotado do que pelo tipo de cobertura superior do café (SAFs). Esta afirmação fica evidenciada nos dendrogramas de dissimilaridade dos 20 tratamentos, nas duas épocas de avaliação, que mostram a formação de grupos bem distintos (Figura 1). Em 2002, observou-se a formação de dois grupos homogêneos, o grupo 1, formado pelos tratamentos com manejo AC, e o grupo 2, formado pelos demais tratamentos. Em 2005, observou-se a formação de três grupos distintos, o grupo 1 , formado por três dos quatro tratamentos $\mathrm{AC}$; $\mathrm{o}$ grupo 2, formado pelos tratamentos E-MC, E-MO, TE-MC, CE-AC e CE-AC, e o grupo 3, formado pelos demais tratamentos.

Com relação à cobertura do solo, pela média dos tratamentos, em 2002, 84,3\% dos pontos se encontravam com invasoras, $7 \%$ com serapilheira e $8,7 \%$ com solo descoberto, enquanto em $2005,57,4 \%$ dos pontos se encontravam com invasoras, $33,8 \%$ com serapilheira e $8,8 \%$ com solo descoberto (Tabela 4). Portanto, ao longo do período avaliado, ocorreu redução da presença de invasoras e aumento dos pontos com serapilheira, mas não houve alteração no número de pontos com solo descoberto.

A influência das árvores sobre a ocorrência de invasoras em cultivos de café foi relatada por outros autores. No México, Nestel \& Altieri (1992) observaram no final do período chuvoso, que nos cafezais a pleno sol a produção de biomassa pelas invasoras foi mais do que o dobro do encontrado no sistema arborizado (sombreado), o que levou esses autores a concluir que a diversificação dos cultivos pode ser uma alternativa para pequenos produtores controlar o surgimento de invasoras. Muschler (1998) observou redução da biomassa de invasoras de $3,6 \mathrm{Mg} \mathrm{ha}^{-1}$ em cultivos de café a pleno sol, para menos de $0,1 \mathrm{Mg} \mathrm{ha}^{-1}$ em cultivos com 50\% ou mais de sombra. Staver et al. (2001) explicam que a quantidade de biomassa de invasoras, presentes em um sistema agroflorestal com café, é extremamente dependente da quantidade de luz filtrada pelas espécies arbóreas e pelo dossel dos cafeeiros presentes nos sistemas.

Nos tratamentos com manejo MC, o tipo de cobertura arbórea foi determinante em 2005, e nos tratamentos com a eritrina, o percentual de invasoras foi sempre menor em comparação aos demais tratamentos com o mesmo manejo (6, 9, 11 e 20) (Tabela 4). Nos tratamentos AC, a cobertura de solo foi influenciada muito mais pela intensidade de manejo, do que pelo tipo de cobertura arbórea, e, nesses tratamentos, foram observados os menores percentuais de invasoras e os maiores valores com solo descoberto. 
A

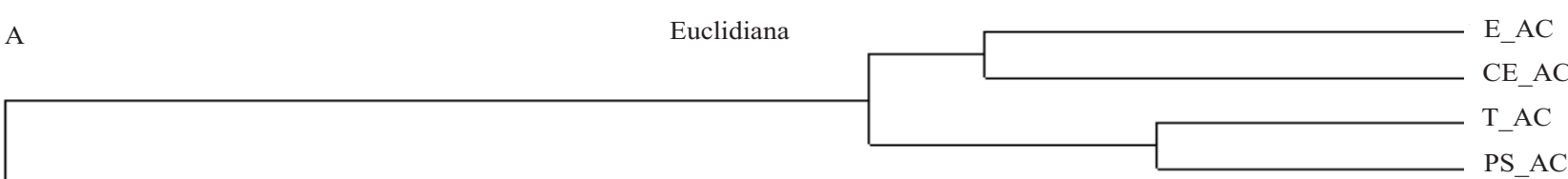

E $\mathrm{MC}$
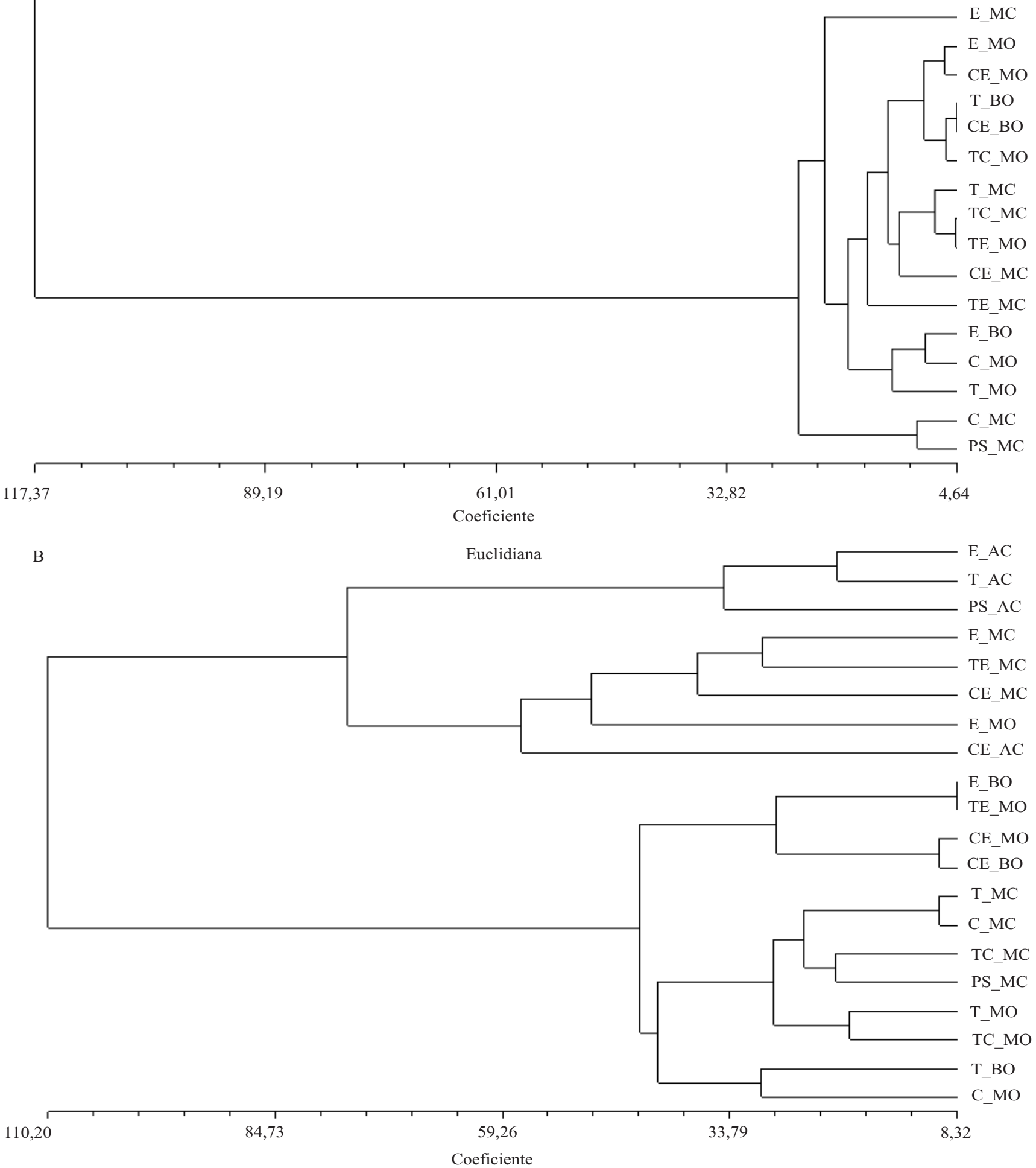

Figura 1. Dendrogramas de dissimilaridade dos tratamentos estudados, em julho de 2002 (A) e setembro de 2005 (B).E: eritrina; T: terminalia; C: cashá; PS: pleno sol; AC: manejo alto convencional; MC: médio convencional; MO: médio orgânico; BO: baixo orgânico. 
Tabela 4. Percentagem de invasoras, solo com serapilheira e solo descoberto dos pontos amostrados pelo método "ponta do sapato" (total de 100 pontos) $)^{(1)}$.

\begin{tabular}{|c|c|c|c|c|c|c|}
\hline \multirow[t]{2}{*}{ Tratamento $^{(2)}$} & \multicolumn{2}{|c|}{ Com invasoras } & \multicolumn{2}{|c|}{ Com serapilheira } & \multicolumn{2}{|c|}{ Solo descoberto } \\
\hline & 2002 & 2005 & 2002 & 2005 & 2002 & 2005 \\
\hline & \multicolumn{6}{|c|}{ Alto convencional } \\
\hline 1 (E-AC) & $13,6 \mathrm{Ac}$ & $8,7 \mathrm{Ac}$ & $52,1 \mathrm{Aa}$ & $62,3 \mathrm{Ab}$ & $34,3 \mathrm{Aa}$ & $29,0 \mathrm{Aa}$ \\
\hline $5(\mathrm{~T}-\mathrm{AC})$ & $33,9 \mathrm{Ab}$ & $3,3 \mathrm{Bc}$ & $20,3 \mathrm{Bb}$ & 78,7Aa & $45,8 \mathrm{Aa}$ & $18,0 \mathrm{Ba}$ \\
\hline 15 (CE-AC) & $18,0 \mathrm{Ac}$ & $4,7 \mathrm{Ac}$ & $38,7 \mathrm{Ba}$ & $68,7 \mathrm{Aa}$ & $43,3 \mathrm{Aa}$ & $26,7 \mathrm{Aa}$ \\
\hline \multirow[t]{2}{*}{19 (PS-AC) } & $48,4 \mathrm{Ab}$ & $18,0 \mathrm{Bc}$ & $15,8 \mathrm{Bb}$ & $44,7 \mathrm{Ab}$ & $35,8 \mathrm{Aa}$ & $37,3 \mathrm{Aa}$ \\
\hline & \multicolumn{6}{|c|}{ Médio convencional } \\
\hline 2 (E-MC) & $92,0 \mathrm{Aa}$ & $23,4 \mathrm{Bb}$ & $5,0 \mathrm{Bb}$ & $74,6 \mathrm{Aa}$ & $6,3 \mathrm{Ab}$ & $2,0 \mathrm{Ac}$ \\
\hline 6 (T-MC) & $100,0 \mathrm{Aa}$ & $83,3 \mathrm{Aa}$ & $0,0 \mathrm{Ab}$ & $11,4 \mathrm{Ac}$ & $0,0 \mathrm{Ab}$ & $5,3 \mathrm{Ac}$ \\
\hline 9 (C-MC) & $100,0 \mathrm{Aa}$ & $83,3 \mathrm{Aa}$ & $0,0 \mathrm{Ab}$ & $8,0 \mathrm{Ac}$ & $0,0 \mathrm{Bb}$ & $8,7 \mathrm{Ab}$ \\
\hline 11 (TC-MC) & $99,3 \mathrm{Aa}$ & $73,7 \mathrm{Ba}$ & $0,0 \mathrm{Ab}$ & $15,0 \mathrm{Ac}$ & $0,7 \mathrm{Bb}$ & $11,3 \mathrm{Ab}$ \\
\hline 13 (TE-MC) & 99,0Аа & $29,7 \mathrm{Bb}$ & $0,7 \mathrm{Bb}$ & $62,0 \mathrm{Ab}$ & $0,3 \mathrm{Bb}$ & $8,3 \mathrm{Ab}$ \\
\hline 16 (CE-MC) & $97,0 \mathrm{Aa}$ & $43,7 \mathrm{Bb}$ & $2,0 \mathrm{Bb}$ & $49,0 \mathrm{Ab}$ & $1,0 \mathrm{Ab}$ & $7,3 \mathrm{Ab}$ \\
\hline \multirow[t]{2}{*}{20 (PS-MC) } & $97,3 \mathrm{Aa}$ & $75,3 \mathrm{Ba}$ & $1,0 \mathrm{Bb}$ & $23,0 \mathrm{Ac}$ & $1,7 \mathrm{Ab}$ & $1,7 \mathrm{Ac}$ \\
\hline & \multicolumn{6}{|c|}{ Médio orgânico } \\
\hline 3 (E-MO) & $96,0 \mathrm{Aa}$ & $39,5 \mathrm{Bb}$ & $2,7 \mathrm{Bb}$ & $57,8 \mathrm{Ab}$ & $1,3 \mathrm{Ab}$ & $2,7 \mathrm{Ac}$ \\
\hline 7 (T-MO) & $100,0 \mathrm{Aa}$ & $77,1 \mathrm{Ba}$ & $0,0 \mathrm{Bb}$ & $19,9 \mathrm{Ac}$ & $0,0 \mathrm{Ab}$ & $3,0 \mathrm{Ac}$ \\
\hline 10 (C-MO) & 99,7Aa & $96,7 \mathrm{Aa}$ & $0,0 \mathrm{Ab}$ & $3,0 \mathrm{Ac}$ & $0,3 \mathrm{Ab}$ & $0,3 \mathrm{Ac}$ \\
\hline 12 (TC-MO) & $100,0 \mathrm{Aa}$ & $86,3 \mathrm{Aa}$ & $0,0 \mathrm{Ab}$ & $13,0 \mathrm{Ac}$ & $0,3 \mathrm{Ab}$ & $0,7 \mathrm{Ac}$ \\
\hline 14 (TE-MO) & 99,7Aa & $72,8 \mathrm{Ba}$ & $0,3 \mathrm{Bb}$ & $23,2 \mathrm{Ac}$ & $0,0 \mathrm{Ab}$ & $4,0 \mathrm{Ac}$ \\
\hline \multirow[t]{2}{*}{17 (CE-MO) } & $98,3 \mathrm{Aa}$ & $79,2 \mathrm{Aa}$ & $0,7 \mathrm{Ab}$ & $19,1 \mathrm{Ac}$ & $0,3 \mathrm{Ab}$ & $1,7 \mathrm{Ac}$ \\
\hline & \multicolumn{6}{|c|}{ Baixo orgânico } \\
\hline $4(\mathrm{E}-\mathrm{BO})$ & $97,4 \mathrm{Aa}$ & $70,0 \mathrm{Ba}$ & $1,3 \mathrm{Bb}$ & $26,6 \mathrm{Ac}$ & $1,3 \mathrm{Ab}$ & $3,4 \mathrm{Ac}$ \\
\hline 8 (T-BO) & $99,3 \mathrm{Aa}$ & $98,0 \mathrm{Aa}$ & $0,0 \mathrm{Ab}$ & $1,0 \mathrm{Ac}$ & $0,7 \mathrm{Ab}$ & $1,0 \mathrm{Ac}$ \\
\hline $18(\mathrm{CE}-\mathrm{BO})$ & $99,7 \mathrm{Aa}$ & $82,0 \mathrm{Aa}$ & $1,3 \mathrm{Ab}$ & $14,7 \mathrm{Ac}$ & $0,3 \mathrm{Ab}$ & $3,3 \mathrm{Ac}$ \\
\hline Média & $84,3 \mathrm{~A}$ & $57,4 \mathrm{~B}$ & $7,0 \mathrm{~B}$ & $33,8 \mathrm{~A}$ & $8,7 \mathrm{~A}$ & $8,8 \mathrm{~A}$ \\
\hline CV tratamentos (\%) & \multicolumn{2}{|c|}{12,3} & \multicolumn{2}{|c|}{56,8} & \multicolumn{2}{|c|}{50,4} \\
\hline CV épocas $(\%)$ & \multicolumn{2}{|c|}{18,1} & \multicolumn{2}{|c|}{55,1} & \multicolumn{2}{|c|}{45,1} \\
\hline
\end{tabular}

Com relação à exposição do solo, os maiores percentuais de pontos com solo descoberto foram encontrados nos quatro tratamentos com manejo AC, em ambas as épocas; nesta intensidade de manejo, os três tratamentos com cobertura arbórea não foram diferentes do tratamento a pleno sol, o que demonstra que quando a aplicação de herbicida é freqüente, a presença de árvores no sistema não garante a cobertura do solo com serapilheira. Além disso, os menores percentuais de solo descoberto, em 2005, foram encontrados em todos os tratamentos com manejo $\mathrm{MO}$ e BO, e em três dos sete tratamentos com manejo MC (Tabela 4).

Embora os parâmetros avaliados tenham sido influenciados pelo tipo de cobertura arbórea e pelo tipo de manejo, a análise de agrupamento demonstrou que o tipo de manejo influenciou mais na separação dos tratamentos em grupos do que o tipo de cobertura arbórea (Figura 1), o que mostra ter ocorrido uma nítida separação dos tratamentos E-AC, T-AC, PS-AC, em ambas as épocas avaliadas, com exceção do tratamento CE-AC em 2002.

\section{Conclusões}

1. Ao longo do tempo, ocorre redução nos valores de riqueza, abundância, diversidade e eqüitabilidade da comunidade de plantas invasoras, em que os menores valores são encontrados nos tratamentos com manejo alto convencional.

2. Com exceção dos tratamentos com manejo alto convencional, a eqüitabilidade é alta, o que significa que os sistemas estudados apresentam distribuição equilibrada das invasoras.

3. Ao longo do tempo, observa-se redução da presença de invasoras e aumento da serapilheira.

4. O tipo e a intensidade de manejo diferencia mais os tratamentos do que o tipo de cobertura arbórea.

\section{Agradecimentos}

Ao Sr. José Luiz Rodrigues Zupanni e à Sra. Maria Fernanda do Prado, respectivamente, dos cursos de Engenharia Florestal e Biologia da Universidade Federal de Lavras, pela coleta dos dados. 


\section{Referências}

AGUILAR, A.; STAVER, C.; AGUILAR, V.; SOMARRIBA, S. Manejo selectivo de malezas para la conservación del suelo en café: evaluación de sistemas químico/mecanico y mecanismo sin y con Arachis pintoi. In: SIMPÓSIO LATINOAMERICANO DE CAFEICULTURA, 18., 1997, San José. Memorias. San José: IICA, 1997. p.85-92.

AGUILAR, V.; SOMARRIBA, S.; STAVER, C. Cinco sistemas de manejo de malezas en café con y sin herbicidas, coberturas sembradas y malezas de cobertura: evaluación preliminar de composición botánica y custos. In: SIMPÓSIO SOBRE CAFEICULTURA LATINOAMERICANA, 16., 1993, Managua. Memoria. Tegucigalpa: IICA: CONCAFE, 1995. v.2. p.190-200.

ALTIERI, M.A. The ecological role of biodiversity in agroecosystems. Agriculture Ecosystems and Environment, v.74, p.19-31, 1999.

ALVARENGA, M.I.N.; MARTINS, M. Fatores edáficos de cafezais arborizados. In: MATSUMOTO, S.N. (Org.). Arborização de cafezais no Brasil. Vitória da Conquista: Uesb, 2004. p.45-84.

ÁVILA, G.; JIMÉNEZ, F.; BEER, J.; GÓMEZ, M.; IBRAHIM, M. Almacenamiento, fijación de carbono y valoración de servicios ambientales en sistemas agroforestales en Costa Rica. Agroforestería en las Américas, v.8, p.32-35, 2001.

BEER, J. Advantages, disadvantages and desirable characteristics of shade trees for coffee, cacao and tea. Agroforestry Systems, v.5, p.3-13, 1987.

BEER, J.; MUSCHLER, R.; KASS, D.; SOMARRIBA, E. Shade management in coffee and cacao plantations. Agroforestry Systems, v.38, p.139-164, 1998.

CAVALCANTI, E.A.H.; LARRAZÁBAL, M.E.L. de. Macrozooplâncton da zona econômica exclusiva do Nordeste do Brasil (segunda expedição oceanográfica - REVIZEE/NE II) com ênfase em Copepoda (Crustacea). Revista Brasileira de Zoologia, v.21, p.467-475, 2004.

DIAS, P.F.; SOUTO, S.M.; RESENDE, A.S.; MOREIRA, J.F.; POLIDORO, J.C.; CAMPELLO, E.F.C.; FRANCO, A.A. Influência da projeção das copas de espécies de leguminosas arbóreas nas características químicas do solo. Pasturas Tropicales, v.28, p.8-17, 2006.

DÍAZ-ROMEÚ, R.; HUNTER, A. Metodología de muestreo de suelos, análisis químico de suelos y tejido vegetal e investigación en invernadero. Turrialba: Catie, 1978. 68p.

ERASMO, E.A.L.; PINHEIRO, L.L.A.; COSTA, N.V. Levantamento fitossociológico das comunidades de plantas infestantes em áreas de produção de arroz irrigado cultivado sob diferentes sistemas de manejo. Planta Daninha, v.22, p.195-201, 2004.

FERREIRA, D.F. Análises estatísticas por meio do SISVAR para Windows versão 4.0. In: REUNIÃO ANUAL DA REGIÃO BRASILEIRA DA SOCIEDADE INTERNACIONAL DE BIOMETRIA, 45., 2000, São Carlos, SP. Programa e resumos. São Carlos: Ufscar, 2000. p.255-258.

FIGUEROA, I.; STAY, M.E.; PLANES, M.; OLIVARES, K. La integración del árbol y del arbusto en la incidencia de malezas en el cultivo del café (Coffea arabica L.). Café Cacao, v.3, p.9-12, 2002.

HAGGAR, J.P.; SCHIBLI, C.; STAVER, C. ¿Cómo manejar árboles de sombra en cafetales? Agroforestería en las Américas, v.8, p.37-41, 2001.

LEVINTON, J.S. Marine biology: function, biodiversity, ecology. New York: Oxford University Press, 1995. 420p.

MAGURRAN, A.E. Ecological diversity and its measurements. Princeton: Princeton University Press, 1988. 179p.

MARTINS, F.R.; SANTOS, F.A.M. Técnicas usuais de estimativa da biodiversidade. Revista Holos, v.1, p.236-267, 1999.

MUSCHLER, R.G. Árborles en cafetales: proyecto agroforestal CATIE/GTZ. Turrialba: CATIE, 2000. 139p. (Módulo de Enseñanza Agroforestal, 5).

MUSCHLER, R.G. Tree-crop compatibility in agroforestry: production and quality of coffee grown under managed tree shade in Costa Rica. 1998. 219p. Thesis (Ph.D.) - University of Florida, Gainesville.

NELSON, D.W.; SOMMER, L.E. Total carbon and organic matter. In: SPARKS, D.L. Methods of soil analysis: part 3: chemical methods. $3^{\text {rd }}$ ed. Madison: ASA; SSSA, 1996. p.961-1110.

NESTEL, D.; ALTIERI, M. The weed community of Mexican coffee agroecosystems: effect of management upon plant biomass and species composition. Acta Oecologica, v.13, p.715-726, 1992.

PAYÁN, F.Z.; BEER, J.; JONES, D.; HARMAND, J.M.; MUSCHLER, R. Concentraciones de carbono y nitrógeno en el suelo bajo Erythrina poeppigiana en plantaciones orgánicas y convencionales de café. Agroforesteria en Las Américas, v.9, p.10-15, 2002.

PELÁEZ, J.J.Z. Dinámica da matéria orgánica del suelo en sistemas agroforestales de café con Erythrina poeppiniana (Walpers) O.F. Cook en Costa Rica. 2004. 116p. Tesis (Mestrado) - Centro Agronómico Tropical de Investigación y Enseñanza, Turrialba.

ROHLF, F.J. NTSYS-pc: numerical taxonomy and multivariate analysis system, version 2.1. New York: Exeter Software, 2000.

SILVA, S.O.; MATSUMOTO, S.N.; BEBÉ, F.V.; SÃO JOSÉ, A.R. Diversidade e frequiência de plantas daninhas em associações entre cafeeiros e grevíleas. Coffee Science, v.1, p.126-134, 2006.

SOTO-PINTO, L.; PERFECTO, I.; CABALLERO-NIETO, J. Shade over coffee: its effects on berry borer, leaf rust and spontaneous herbs in Chiapas, México. Agroforestry Systems, v.55, p.37-45, 2002.

STAVER, C. ¿Cómo tener más hierbas de cobertura y menos malezas en nuestros cafetales? Agroforestería en las Américas, v.8, p.30-32, 2001.

STAVER, C.; GUHARAY, F.; MONTERROSO, D.; MUSCHLER, R.G. Designing pest-suppressive multistrata perennial crop systems: shade-grown coffee in Central America. Agroforestry Systems, v.53, p.151-170, 2001.

YANAGIZAWA, Y.A.N.P.; MAIMONI-RODELLA, R.C.S. Composição florística e estrutura da comunidade de plantas do estrato herbáceo em áreas de cultivo de árvores frutíferas. Planta Daninha, v.17, p.459-468, 1999. 